\title{
Lombalgia gestacional: prevalência e características de um programa pré-natal
}

\author{
Low back pain in pregnancy: prevalence and characteristics of a prenatal program
}

Marília Manfrin dos Santos', Ana Paula Gallo,

\begin{abstract}
Resumo
Introdução: A dor lombar é uma queixa comum na população em geral e é sintoma frequentemente relacionado à gestação. Esse sintoma é limitante, já que interfere diretamente nas atividades de vida diária (AVD) e qualidade de vida. Objetivo: Determinar a prevalência de lombalgia e descrever suas principais características em gestantes. Métodos: Quarenta e cinco gestantes do primeiro ao terceiro trimestre de gestação, assistidas pelo Programa de Pré-Natal do Ambulatório Municipal João Lânia, em José Bonifácio (SP), foram selecionadas para este estudo observacional. As pacientes foram entrevistadas e os dados referentes ao tipo, intensidade, frequência e duração da dor lombar, início dos sintomas, presença de lombalgia prévia, fatores agravantes, influência nas AVDs e prática de atividade física foram obtidos pela aplicação de um questionário direto. Resultados: A prevalência de dor lombar foi de $73 \%$. A lombalgia foi mais frequente em mulheres que apresentaram lombalgia prévia $(p<0,01)$ e naquelas que estiveram grávidas pela primeira vez $(p=0,05)$. A dor lombar também foi mais frequente durante o terceiro trimestre gestacional $(52 \%)$ e, na maioria dos casos, foi referida como pontadas, de intensidade moderada, com duração de uma hora ou mais. Os sintomas ocorriam especialmente à tarde (67\%), com piora no período da noite (64\%). 0 repouso reduzia a dor lombar em $70 \%$ das pacientes, enquanto as atividades domésticas intensas a agravavam em $64 \%$. Conclusões: A lombalgia é um achado comum em gestantes, com características específicas e fatores agravantes que podem ser facilmente identificados e tratados durante a rotina pré-natal.
\end{abstract}

Palavras-chave: Dor lombar; gravidez; prevalência.

\begin{abstract}
Introduction: Low back pain is a common complaint in general population and it is a frequent symptom related to pregnancy. This symptom is limiting, because it interferes directly in daily living activities (DLA) and in quality of life. Objective: The aim of this study was to determinate the low back pain prevalence and to describe its main characteristics among pregnant women. Methods: Forty-five pregnant women from the first to the third trimester of pregnancy, assisted by the Prenatal Program of the Ambulatório Municipal João Lânia, in José Bonifácio, São Paulo, Brazil, were enrolled in this observational study. Patients were interviewed and the data regarding type, intensity, frequency and duration of low back pain, the beginning of the symptoms, presence of previous low back pain, worsening factors, influence in DLA and physical activity practice were obtained by means of application of a directed questionnaire. Results: The prevalence of low back pain was 73\%. Low back pain was more frequent in women who experienced previous low back pain $(p<0,01)$ and in those who were pregnant for the first time $(p=0,05)$. Low back pain was also more frequent during the third trimester of pregnancy $(52 \%)$ and, in most cases, was referred as twinges with moderate intensity, lasting one or more hours. The symptoms occurred mainly in the afternoon (67\%) and became worse at night (64\%). Rest was found to alleviate the symptoms in $70 \%$ of the patients, whereas intensive domestic activities made them worse in $64 \%$. Conclusions: Low back pain is a common finding in pregnant women, with specific characteristics and worsening factors that could be easily identified and addressed during prenatal routine.
\end{abstract}

Keywords: Low back pain; pregnancy; prevalence.

Recebido: $13 / 7 / 2009$

Revisado: $11 / 9 / 2009$

Aprovado: $24 / 5 / 2010$

Universidade Paulista Unip (Unip), São José do Rio Preto (SP), Brasil

2 Academia de Ciências e Tecnologia, São José do Rio Preto (SP), Brasil

Trabalho realizado no Ambulatório Municipal João Lânia, José Bonifácio (SP), Brasil

Endereço para correspondência: Ana Paula Gallo - Academia de Ciência e Tecnologia - Rua Bonfá Natale, 1.860 - Santos Dumont - São José do Rio

Preto (SP) - CEP 15020-130 - E-mail: anapgallo@yahoo.com.br 


\section{Introdução}

A gravidez é um período no qual ocorrem alterações físicas e emocionais, com o intuito de adaptar a mulher à sua nova condição de gestante ${ }^{1}$. Entre essas modificações estão alterações hormonais, musculoesqueléticas, cardiovasculares, respiratórias, tegumentares, nervosas, gastrointestinais e urogenitais ${ }^{2}$.

Do ponto de vista biomecânico, ocorre um deslocamento do centro de gravidade para frente, devido ao aumento do abdome e das mamas, o que leva a alterações de postura, como diminuição do arco plantar, hiperextensão dos joelhos e anteversão pélvica. Esses ajustes geram uma acentuação da lordose lombar e consequente tensão da musculatura paravertebral ${ }^{2}$. A partir do segundo trimestre gestacional, a sobrecarga nos músculos e ligamentos da coluna vertebral é ainda mais intensa, devido à ação de hormônios como o estrogênio e a relaxina sobre os grandes ligamentos das articulações pélvicas ${ }^{3,4}$.

A dor lombar ou lombalgia é um sintoma frequente em gestantes e acomete cerca de $50 \%$ das mulheres 5 . Sua etiologia está ligada a inúmeros fatores, tais como as alterações fisiológicas, biomecânicas, vasculares e psicológicas ${ }^{6}$. Segundo Ostgaard et al., a lombalgia gestacional pode ser classificada em dor lombar, dor pélvica posterior e na combinação das duas anteriores. Clinicamente, a dor lombar é uma condição que se caracteriza por dor à palpação da musculatura paravertebral, diminuição da amplitude de movimento da coluna lombar, interferindo pouco na marcha e na postura. Por outro lado, a dor pélvica posterior tem caráter intermitente, irradia-se para a região glútea, piorando com a marcha e a postura estática ${ }^{7}$. A lombalgia gestacional é um sintoma limitante, pois interfere nas atividades de vida diária (AVDs) e na qualidade de vida. De acordo com Novaes et al. ${ }^{5}$, os sintomas da lombalgia podem durar até três anos após a gestação e ocorrem, principalmente, no último trimestre gestacional.

O tratamento de escolha para lombalgia é o uso de anti-inflamatórios, analgésicos e fisioterapia. Além dessas abordagens, é necessário esclarecer quanto às medidas preventivas, tais como hábitos posturais, sono e prática regular de exercícios físicos ${ }^{5}$. Em gestantes, a fisioterapia contribui para o alívio da dor e melhora na qualidade de vida ${ }^{1,8}$. Estudo de Moura et al., com 14 gestantes, evidenciou que um protocolo de exercícios ativos de alongamento, fortalecimento e relaxamento, realizado por 3 meses consecutivos, contribuiu para redução da intensidade da dor em mulheres com lombalgia gestacional ${ }^{8}$. Outros métodos, como stretching global ativo, massoterapia, acupuntura e hidroterapia, também têm se mostrado eficazes ${ }^{9-12}$. O guideline europeu, publicado em 2008, recomenda que o tratamento da gestante com dor pélvica seja individual e que envolva mais de uma modalidade de intervenção terapêutica ${ }^{13}$.

O presente trabalho teve como objetivo detectar a prevalência de lombalgia gestacional e descrever as principais características desse sintoma em gestantes acompanhadas em um programa pré-natal.

\section{Casuística e métodos}

Para avaliar a prevalência e as características da lombalgia em gestantes, foi conduzido um estudo transversal.

Foi utilizada uma amostra não-probabilística por conveniência, na qual todas as gestantes do Programa de Pré-Natal do Ambulatório Municipal João Lânia da cidade de José Bonifácio (SP), no período de 15 de agosto a 30 de setembro de 2008, participaram do estudo. O curto período serviu ao propósito do estudo, pois, dessa forma, foi possível selecionar a quase totalidade das gestantes atendidas no ambulatório, já que o agendamento das consultas pré-natais era mensal. Os critérios de exclusão foram: diagnóstico de gestação de alto risco, tratamento fisioterápico para lombalgia no momento da entrevista, presença de deficiência cognitiva/mental e não comparecimento à entrevista. $\mathrm{O}$ presente estudo foi aprovado pelo Comitê de Ética e Pesquisa da Faculdade de Medicina de São José do Rio Preto (Famerp) - parecer $n^{\circ}$ $462 / 2008$ - e as participantes assinaram um termo de consentimento livre e esclarecido.

Os dados foram coletados em salas de espera, antes da consulta médica, por meio de entrevista, na qual as perguntas foram enunciadas pela entrevistadora e preenchidas pela mesma com as respostas do pesquisado. Foi criado e aplicado um questionário baseado em Assis e Tibúrcio $^{14}$, composto por 17 perguntas simples, com respostas fechadas referentes às características da dor lombar. Esse questionário permitiu verificar o tipo, intensidade, frequência e duração da dor lombar, início dos sintomas durante a gestação, presença de dor lombar prévia à gestação, fatores que agravam e que amenizam o sintoma, influência da dor nas AVDs, atividades domésticas e sono, tipos de orientações recebidas quanto à dor lombar e a prática regular de atividade física antes e durante o período gestacional. Para avaliar a intensidade da dor, foi utilizada a Escala Visual Numérica de Dor, na qual a paciente recebia a escala impressa e apontava um número que correspondia ao sintoma referido, em que: 0 significava ausência de dor; 1 a 3, dor leve; 4 a 6 , dor moderada; e 7 a 10 correspondia a dor grave.

$\mathrm{O}$ teste $t$ não-pareado foi utilizado para comparar médias de idade e do índice de massa corpórea (IMC) entre as gestantes com e sem lombalgia. O teste do $\chi^{2}$ foi utilizado para avaliar diferenças nos grupos de gestantes com e sem lombalgia em relação às seguintes variáveis qualitativas: gestação prévia (primíparas ou multíparas) e dor lombar prévia (sim ou não). A diferença foi considerada significativa quando $\mathrm{p} \leq 0,05$.

\section{Resultados}

Participaram do estudo 45 gestantes, entre o primeiro e terceiro trimestre de gestação, primíparas e multíparas, com idades entre 13 e 38 anos. A média de idade das gestantes entrevistadas foi de 26 anos, sendo que $51 \%$ eram pardas, $36 \%$ brancas e $13 \%$ negras. A grande maioria era casada $(77 \%)$ e todas eram alfabetizadas $(64 \%)$ ou semialfabetizadas 
Tabela1 - Média de idade e índice de massa corpórea em gestantes com e sem lombalgia

\begin{tabular}{lcrc}
\hline Gestantes & Com lombalgia & Sem lombalgia & Valor de $p$ \\
\hline Média de idade (anos) & $24,2( \pm 5,5)$ & $27,6( \pm 5,4)$ & 0,07 \\
Média do IMC & $27,1( \pm 5)$ & $24,9( \pm 3,2)$ & 0,16 \\
\hline
\end{tabular}

IMC: índice de massa corpórea.

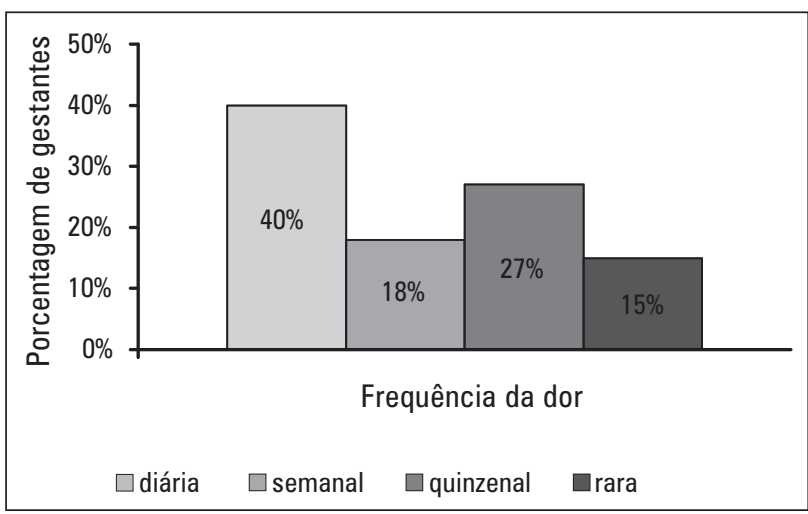

Figura 1 - Intensidade da dor lombar na gestação.

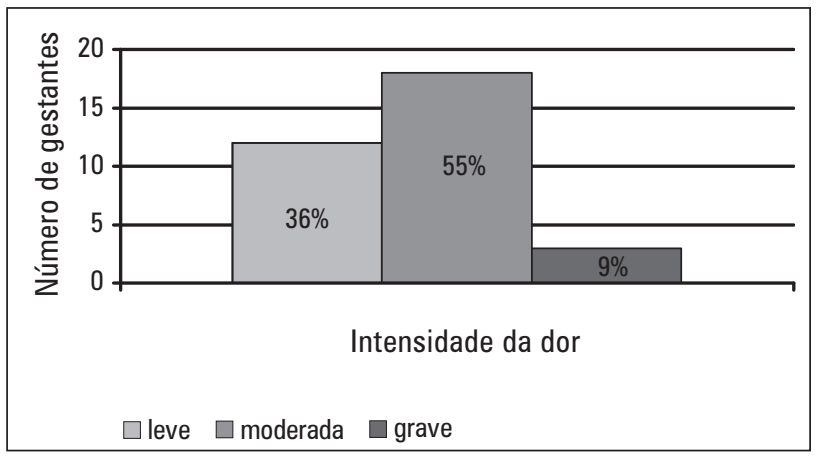

Figura 2 - Frequência da dor em gestantes com lombalgia.

(36\%). As primíparas, ou seja, mulheres que não haviam passado por nenhum parto, corresponderam a $45 \%$ da casuística, enquanto que as multíparas, isto é, mulheres que haviam dado à luz uma ou mais vezes, somaram 55\%. Quanto à ocupação, $64 \%$ mulheres eram do lar, o que não influenciou a presença de lombalgia durante a gestação $(p=0,85)$.

No presente estudo, a prevalência de lombalgia gestacional foi de $73 \%$. No grupo de gestantes com dor lombar, esta foi mais frequente no último trimestre gestacional (48\%), seguido do segundo trimestre (43\%).

Os dados referentes à idade e ao IMC das gestantes com e sem lombalgia podem ser visualizados na Tabela 1. As médias de idade e IMC não foram diferentes entre as gestantes com e sem lombalgia ( $\mathrm{p}=$ $0,07 \mathrm{e} \mathrm{p}=0,16$, respectivamente) (Tabela 1$)$.

A análise das variáveis qualitativas revelou que a prevalência de lombalgia foi maior nas gestantes primíparas em comparação com as multíparas $(\mathrm{p}=0,05) ; 60 \%$ das gestantes multíparas apresentavam lombalgia gestacional, enquanto $90 \%$ das primíparas apresentavam essa complicação. Da mesma forma, a prevalência de lombalgia gestacional foi maior nas mulheres com antecedente de dor lombar em comparação com aquelas sem histórico de lombalgia prévia $(\mathrm{p}=0,0002)$. Episódios prévios de dor lombar foram relatados por $85 \%$ das gestantes com lombalgia gestacional.

A avaliação da intensidade da dor no grupo de gestantes com lombalgia pode ser visualizada na Figura 1. Ainda nesse grupo, 54\% das entrevistadas relataram aumento da intensidade da dor com o avanço da gestação, sendo que a maioria referiu dor como pontada (48\%) e como queimação (40\%).

Em 48\% das gestantes com lombalgia, a dor permanecia localizada na região da coluna lombar, enquanto que, em $52 \%$ dos casos, a dor irradiava para outras regiões do corpo, principalmente para as pernas e coxas (33\%) e para o abdome (18\%).

Quanto à frequência da dor no grupo com lombalgia, verificou-se que, em $40 \%$ das gestantes, o sintoma ocorria diariamente e apenas em $15 \%$ a dor foi considerada rara (Figura 2). A dor era sentida principalmente no período da tarde (67\%), com agravo dos sintomas à noite (64\%).

Em relação à duração da dor lombar nas gestantes sintomáticas, apenas $12 \%$ referiram senti-la por menos de uma hora, contrastando com $88 \%$ das mulheres que sentiam dor lombar por uma hora ou mais. Para aliviar o sintoma doloroso, a atitude mais adotada foi o repouso (70\%), seguido de massagens no local (12\%) e exercícios específicos $(9 \%)$. Os principais fatores agravantes da dor lombar foram atividades domésticas, como lavar e passar a roupa e limpar a casa (64\%), permanência sentada ou em pé por muito tempo (30\%) e vícios posturais $(3 \%)$.

No que diz respeito ao nível de atividade física, verificou-se que, das gestantes com lombalgia, $21 \%$ praticavam atividade física regular durante a gravidez, sendo que $79 \%$ não praticavam nenhum exercício físico regular. Cabe ressaltar que duas mulheres iniciaram um programa de exercício físico após a gestação, enquanto seis deixaram de se exercitar durante o período gestacional.

A dor lombar influenciou as AVDs da maior parte das gestantes com lombalgia. Apesar de $45 \%$ não relatarem piora da dor ao executar atividades domésticas, tais como varrer a casa ou lavar e passar a roupa, $55 \%$ relataram que já sentiram dor insuportável e que não puderam executar essas atividades ou mesmo ir ao trabalho. Metade dessas mulheres (51\%) já acordou à noite por incômodo da dor lombar.

Foi observado que a maioria das gestantes (57\%) não recebeu qualquer tipo de orientação quanto aos cuidados em relação à prevenção da dor lombar durante a gestação. Das mulheres que receberam informações, $71 \%$ as receberam do médico. As orientações mais frequentes foram: repousar (29\%), evitar ficar em pé por muito tempo (6\%) e evitar carregar peso (3\%).

\section{Discussão}

A dor lombar é uma queixa clínica comum na população geral e é considerada um sintoma frequente e habitual entre gestantes, principalmente nos últimos meses. Durante esse período, a maioria das gestantes 
refere dor na coluna vertebral, especialmente na região lombar. O presente estudo constatou que a prevalência de lombalgia gestacional foi de $73 \%$, o que condiz com dados da literatura brasileira, cuja porcentagem varia de 48 a $83 \% \%^{5,9,15}$.

Em estudos prospectivos ${ }^{16,17}$, que compararam a lombalgia em gestantes e não gestantes, verificaram que a prevalência de dor lombar foi maior em gestantes, especialmente a partir do terceiro trimestre. Além disso, foi evidenciado que o aparecimento de lombalgia era comum no período da tarde e à noite. Da mesma forma, os dados deste trabalho corroboram o estudo anterior, pois a dor lombar se iniciou à tarde e se intensificou à noite na maioria das gestantes, sendo mais frequente nos últimos trimestres gestacionais.

Estudo americano que avaliou a severidade da lombalgia gestacional em 645 mulheres revelou que a intensidade da dor lombar foi relatada como moderada na maioria das vezes ${ }^{18}$. Assis e Tibúrcio $^{14}$ constataram que, em relação à percepção da dor, esta foi referida como pontada e aperto, com duração superior a três horas em 37\% dos casos. Da mesma forma, o presente estudo revelou dor em pontada e em queimação e raramente cessava em período inferior a uma hora. Além disso, em mais da metade da casuística, a intensidade da dor aumentou com o tempo de gestação, o que era esperado com o crescimento do útero e sobrecarga na coluna lombar. Embora estudo de Wang et al. ${ }^{18}$ tenha mostrado que a prevalência de lombalgia não foi afetada pela idade gestacional, Assis e Tibúrcio ${ }^{14}$ relataram que, em $60 \%$ dos casos, esse fato ocorria.

Em trabalho conduzido com 203 gestantes participantes de um programa de pré-natal, observou-se que $50 \%$ das gestantes com lombalgia apresentavam sintomas de acometimento nervoso ${ }^{15}$, o que gerava muito incômodo. Na maioria dos casos, a dor irradiava para a região dos glúteos e pernas ${ }^{14}$. Em conformidade, no presente estudo a maioria das gestantes relatou dor irradiada para os membros inferiores.

Sabe-se que a atividade física programada de intensidade leve à moderada proporciona benefícios à gestante, como melhora na eficiência cardíaca e pulmonar, manutenção do peso e composição corpórea em níveis adequados, melhora da força e/ou resistência muscular, melhora da elasticidade muscular, aumento da resistência óssea, prevenção de problemas posturais e redução do estresse ${ }^{19}$. Nesses casos, o exercício físico contribui para uma melhora na adaptação à nova postura física, refletindo-se em maior habilidade para a gestante durante a prática da atividade física e do trabalho diário ${ }^{20}$. Das mulheres avaliadas, $79 \%$ não praticavam nenhum tipo de atividade física, o que também pode ter contribuído para a alta prevalência de lombalgia gestacional. Muitas gestantes continuaram com as atividades domésticas, como lavar a roupa e limpar o chão, o que pode ter contribuído para o aparecimento ou a piora da lombalgia, pois é sabido que o esforço físico não controlado e em excesso aumenta em 2,4 vezes a incidência de lombalgia em gestantes $^{16}$. Em trabalhadoras grávidas, foi verificado que a adoção de pausas para o descanso durante a jornada de trabalho reduz a severidade da dor lombar ${ }^{21}$.
Entre os fatores de risco associados ao desenvolvimento de dor lombar em gestantes estão a idade, a dor lombar prévia e o IMC ${ }^{18,22}$. Sabe-se que quanto mais jovem a gestante, maior a chance de desenvolver lombalgia gestacional ${ }^{15}$. Em conformidade, o presente estudo evidenciou que a queixa de lombalgia durante a gestação ocorreu em mulheres mais jovens. Ainda, no presente trabalho, a frequência de lombalgia gestacional foi maior em mulheres que apresentaram dor lombar prévia, sendo este um dos principais fatores associados a essa queixa durante a gestação. Já foi relatado que, em mulheres que apresentam lombalgia prévia à gestação, existe uma intensificação dos sintomas e estes podem interferir nas $\mathrm{AVDs}^{20}$.

Em aproximadamente $50 \%$ das gestantes, a lombalgia é de intensidade e persistência suficientes para atingir e, muitas vezes, modificar o seu estilo de vida de alguma forma e, para um terço delas, essa dor é de caráter muito grave, interferindo em suas atividades diárias e profissionais $^{5,23}$. Trabalho de Olsson e Nilsson-Wikmar ${ }^{24}$ com 160 gestantes constataram que mais da metade das mulheres com lombalgia, entre a $34^{\mathrm{a}}$ e a $37^{\mathrm{a}}$ semana gestacional, têm suas habilidades físicas prejudicadas, o que interfere diretamente na qualidade de vida. O presente estudo evidenciou que houve influência negativa da dor nas atividades domésticas realizadas pela maior parte das gestantes. Além disso, foi evidenciado que metade das gestantes possuía problemas com o sono, já que, às vezes, eram acordadas à noite devido à dor lombar. A lombalgia noturna está presente em um terço das mulheres com dor lombar e é possível que ocorra pela fadiga muscular acumulada durante o dia ou pela redução do fluxo sanguíneo para a região ${ }^{25}$. Estudo retrospectivo também constatou que 58\% das gestantes apresentavam distúrbios do sono, que interferiam nas $\mathrm{AVDs}^{18}$.

Cabe ressaltar, ainda, que na equipe de pré-natal em que foi realizado este estudo não havia nenhum fisioterapeuta. A presença desse profissional é fundamental em equipes de pré-natal para melhor avaliação, manejo e tratamento da lombalgia, já que a fisioterapia é considerada um tratamento seguro e eficaz na redução de dor lombar durante a gestação $0^{5,8}$.

É de extrema importância o esclarecimento às gestantes em relação aos fatores agravantes e de alívio da lombalgia, ao passo que já é sabido que alguns desconfortos decorrentes da dor lombar podem permanecer por até três anos após o parto ${ }^{5}$. Com os resultados deste trabalho, constatou-se que a grande maioria das gestantes entrevistadas não recebeu orientação sobre o que fazer para prevenir ou minimizar o sintoma lombar, e isso pode ter contribuído para o agravamento da dor. Wang et al. ${ }^{18}$ observaram, em seu estudo com 950 gestantes, que somente 32\% das que apresentavam lombalgia receberam informações sobre os cuidados pré-natal para dor lombar e apenas $25 \%$ dos profissionais de Saúde que as acompanhavam recomendaram tratamento para esse sintoma.

Os fatores que melhoraram a dor lombar na maioria das gestantes foram o repouso e a massoterapia. $\mathrm{O}$ repouso gera um relaxamento dos músculos esqueléticos, o que contribui muito para a melhora da dor. A massoterapia segue o mesmo princípio de relaxamento, associado, ain- 
da, a outros efeitos físicos e psicológicos. Pode-se utilizar a massoterapia clássica, as técnicas miofasciais ou do ponto-gatilho ${ }^{26}$. Estudo de Field et $a l .^{10}$, em gestantes depressivas, demonstrou uma diminuição da dor lombar, da ansiedade e da depressão em mulheres que receberam massoterapia a partir da $20^{\text {a }}$ semana de gestação. Um dos fatores agravantes da lombalgia neste trabalho foi a realização de atividades domésticas. Aguiar et $a l .{ }^{27}$ não verificaram correlação entre lombalgia e idade gestacional em estudo prospectivo com 163 gestantes; entretanto, foi possível notar que houve uma intensificação da dor com a realização de atividades domésticas. Isso se explica pela sobrecarga imposta associada ao fato de ocorrerem modificações durante a gestação que afetam o funcionamento habitual de todos os sistemas corporais, incluindo a biomecânica corporal ${ }^{8}$.
Portanto, conclui-se que a prevalência de lombalgia foi alta na população estudada e predominante nos últimos meses de gestação. Os principais fatores associados à lombalgia foram dor prévia à gestação e primiparidade. A dor lombar caracterizou-se por ser frequente, de intensidade moderada, com duração superior a uma hora, iniciando à tarde e com piora à noite. Além disso, a lombalgia interferiu no sono e nas atividades domésticas, o que sugere uma influência real na qualidade de vida.

Com base nas características da lombalgia gestacional que foram apresentadas pelo presente trabalho, sugere-se a realização de novos estudos, a fim de se estabelecer uma abordagem preventiva e terapêutica eficaz nessa população.

\section{Referências}

1. Kisner C, Colby LA. Exercícios terapêuticos: fundamentos e técnicas. $4^{\mathrm{a}}$ ed. São Paulo: Manole; 2005.

2. Souza ELBL. Fisioterapia aplicada a Obstetrícia: aspectos de ginecologia e neonatologia. $3^{\mathrm{a}}$ ed. Rio de Janeiro: Medsi; 2002.

3. Ostgaard HC. Assessment and treatment of low back pain in working pregnant women. Semin Perinatol. 1996;20(1):61-9.

4. Pennick VE, Young G. Interventions for preventing and treating pelvic and back pain in pregnancy. Cochrane Database Syst Rev. 2007; (2):CD001139.

5. Novaes FS, Shimo AKK, Lopes MHBM. Lombalgia na gestação. Rev Latinoam Enfermagem. 2006;14(4):620-4.

6. Ferreira $\mathrm{CHJ}$, Nakano AMS. Reflexões sobre as bases conceituais que fundamentam a construção do conhecimento acerca da lombalgia na gestação. Rev Latino-am Enfermagem. 2001;9(3):95-100.

7. Ostgaard $\mathrm{HC}$, Andersson GB, Karlsson K. Prevalence of back pain in pregnancy. Spine (Phila Pa 1976). 1991;16(5):549-52.

8. Moura SRV, Campos SR, Mariani SHV, Siqueira AAF, Abreu LC. Dor lombar gestacional: impacto de um protocolo de fisioterapia. Arq Med ABC. 2007:32(Supl. 2):S59-63

9. Martins RF, Silva JLP. Tratamento da lombalgia e dor pélvica posterior na gestação por um método de exercícios. Rev Bras Ginecol Obst. 2005;27(5):275-82

10. Field T, Figueiredo B, Hernadez-Reif M, Diego M, Deeds O, Ascencio A. Massage therapy reduces pain in pregnant women, alleviates prenatal depression in both parents and improves their relationships. J Bodyw Mov Ther. 2008;12(2):146-50.

11. Elden $\mathrm{H}$, Ostgaard HC, Fagevik-Olsen M, Ladfors L, Hagberg H. Treatments of pelvic girdle pain in pregnant women: adverse effects of standard treatment, acupuncture and stabilising exercises on the pregnancy, mother, delivery and the fetus/neonate. BMC Complement Altern Med. 2008;8:34.

12. Pitangui ACR, Ferreira CHJ. Avaliação fisioterapêutica e tratamento da lombalgia gestacional. Fisioter Mov. 2008;21(2):135-42.
13. Vleeming A, Albert HB, Ostgaard HC, Sturesson B, Stuge B. European guidelines for the diagnosis and treatment of pelvic girdle pain. Eur Spine $\mathrm{J}$. 2008;17(6):794-819.

14. Assis RG, Tibúrcio RES. Prevalência e características da lombalgia na gestação: um estudo entre gestantes assistidas no programa de pré-natal da maternidade dona Íris em Goiânia. Trabalho de conclusão de curso. Universidade Católica de Goiás. Goiânia; 2004. p.10-28.

15. Martins RF, Silva JLP. Prevalência de dores nas costas na gestação. Rev Assoc Med Bras. 2005;51(3):144-7.

16. Cecin HA, Bichuetti JAN, Daguer MK, Pustrelo MN. Lombalgia e gravidez. Rev Bras Reumatol. 1992;32(2):45-50.

17. Mann L, Kleinpaul JF, Teixeira CS, Konopka CK. Dor lombo-pélvica e exercício físico durante a gestação. Fisioter Mov. 2008;21(2):99-105.

18. Wang SM, Dezinno P, Maranets I, Berman MR, Caldwell-Andrews AA Kain ZN. Low back pain during pregnancy: prevalence, risk factors, and outcomes. Obstet Gynecol. 2004;104(1):65-70.

19. Lamezon AC, Patriota ALVF. Eficácia da fisioterapia aquática aplicada a gestantes para prevenção e tratamento da lombalgia - revisão sistemática. Terra e Cultura. 2005;21(41):127-32.

20. Batista DC, Chiara VL, Gugelmin SA, Martins PD. Atividade física e gestação: saúde da gestante não atleta e crescimento fetal. Rev Bras Saúde Mater Infant. 2003;3(2):151-8.

21. Cheng PL, Pantel M, Smith JT, Dumas GA, Leger AB, Plamondon A et al. Back pain of working pregnant women: identification of associated occupational factors. Appl Ergon. 2009;40(3):419-23.

22. Mogren IM, Pohjanen AI. Low back pain and pelvic pain during pregnancy: prevalence and risk factors. Spine (Phila Pa 1976). 2005;30(8): 983-91.

23. Verner CM, Verner CM, Barbosa GG, Oliveira MM, Martins FM, Silva CCV. Análise dor lombar nas atividades de vida diária entre gestantes primigestas e multigestas mediante a aplicação do questionário Oswestry, Muriaé - MG: estudo piloto. Rev Cient Faminas. 2007;3(1):105. 
24. Olsson C, Nilsson-Wikmar L. Health-related quality of life and physical ability among pregnant women with and without back pain in late pregnancy. Acta Obstet Gynecol Scand. 2004;83(4):351-7.

25. Fast A, Shapiro D, Ducommun EJ, Friedmann LW, Bouklas T, Floman Y. Lowback pain in pregnancy. Spine (Phila Pa 1976). 1987;12(4):368-71.
26. Braun MB, Simonson SJ. Massoterapia. São Paulo: Manole; 2007.

27. Aguiar EOG, Pereira JS, Silva MAG. Freqüência de dor lombar em grávidas e relação com a idade gestacional. Fisioter Bras. 2007;8(1):31-5. 